\title{
Epidemiological trends of low back pain at the global, regional, and national levels
}

\author{
Linfeng Wang ${ }^{1} \cdot$ Hong Ye ${ }^{1} \cdot{\text { Zhichao } \mathrm{Li}^{2} \cdot \text { Chengwu } \mathrm{Lu}^{1} \cdot \text { Jian Ye }^{1} \cdot \text { Mingxin Liao }}^{1} \cdot$ Xiaojie Chen $^{1}$ (1)
}

Received: 12 September 2021 / Revised: 16 January 2022 / Accepted: 25 January 2022 / Published online: 26 February 2022

(c) The Author(s) 2022

\begin{abstract}
Purpose We aimed to assess the global, regional, and national burdens of low back pain (LBP) from 1990 to 2019 by gender, age, and the sociodemographic index (SDI) from the Global Burden of Disease (GBD) 2019 study.

Methods The number of incident cases, disability-adjusted life years (DALYs), age-standardized incidence rates, and agestandardized DALY rates during 1990-2019 were obtained from the GBD 2019 study. The estimated annual percentage changes (EAPCs) in the age-standardized incidence rates and age-standardized DALY rates were determined to measure the temporal trends of LBP.

Results In 2019, there were an estimated 223.5 million cases of LBP and 63.7 million LBP-related DALYs worldwide. During 1990-2019, the age-standardized incidence rate [EAPC $=-0.41 ; 95 \%$ confidence interval $(\mathrm{CI})-0.46$ to -0.36 ] and age-standardized DALY rate decreased (EAPC $=-0.51 ; 95 \% \mathrm{CI}-0.56$ to -0.46$)$ globally. The age-standardized incidence rate of LBP decreased the most in low-middle SDI regions. The age-standardized incidence rate of LBP decreased the most in South Asia (EAPC, - 1.51), East Asia (EAPC, - 0.68), and Australasia (EAPC, - 0.26). The incidence in male subjects was lower than that in female subjects. The largest decreases in the age-standardized incidence rate and age-standardized DALY rate of LBP were observed in India, and China.

Conclusions The global age-standardized incidence rate and age-standardized DALY rate of LBP showed a downward trend, especially in East and South Asia. In addition, a heavier burden of LBP was observed in older and female populations.
\end{abstract}

Keywords Low back pain · Epidemiological · Trends · Global burden of disease

\section{Introduction}

Although low back pain (LBP) is common in the general adult population, it is usually regarded as a disease with a small burden [1]. However, LBP is one of the major causes of years lived with disability in $65 \%$ of countries globally and the primary cause of years lived with disability in all

Linfeng Wang and Hong Ye these are authors share first authorship.

Xiaojie Chen

fjsnasdyyy@163.com

1 Department of Orthopedics, The Affiliated Nanping First Hospital, Fujian Medical University, 317 Zhongshan Road, Yanping District, Nanping City, Fujian Province, People's Republic of China

2 Department of Orthopedics, Shaowu Municipal Hospital of Fujian Province, Shaowu City, Fujian Province, People's Republic of China high-income countries [2]. Additionally, from 1990 to 2015, the years lived with disability caused by $54 \%$, with the largest increase evidenced in low- and middle-income countries [3].

The GBD 2019 study assesses the burden of 369 human diseases and injuries in 204 countries and territories worldwide [2]. However, there has not been a systematic up-todate of the temporal trends of LBP since the GBD 2019 study. Therefore, we assessed the LBP burden based on the GBD 2019 data by determining the temporal trends in LBP incidence rates and LBP-related disability-adjusted life years (DALYs) at the global, regional, and national levels in terms of sex, age, and the socio-development index (SDI). 


\section{Methods}

\section{Overview}

The GBD 2019 study estimated the incidence and mortality of 369 diseases and injuries and the related DALYs in both sexes and 204 countries and territories, grouping the data by 21 regions and seven super-regions [2]. We obtained the annual incident cases, DALYs, age-standardized incidence rates, and age-standardized DALY rates of LBP from 1990 to 2019 [2]. GBD 2019 includes a great number of data, such as surveys, censuses, vital statistics, and other health-related data sources. The data are applied to calculate the incidence, and disability-adjusted life years (DALYs) for 204 countries and territories from 1990 to 2019. The specific data can be found through the tool: http://ghdx.healthdata.org/gbd-2019/ data-input-sources. The description of the GBD 2019 study are published elsewhere [2].

The SDI, a comprehensive measurement of educational level, income per capita, and fertility rate, is scored from 0 to 1 [4]. The 204 countries and territories included in the study were classified into high, high-middle, middle, low-middle, and low SDI regions and 21 geographical regions [2]. The GBD 2019 study complied with the Guidelines for Accurate and Transparent Health Estimates Reporting (GATHER) Guidelines [5, 6]. The Affiliated Nanping First Hospital of Fujian Medical University reviewed and approved this study.

\section{Estimation framework}

In GBD 2019 study, LBP is defined as LBP with or without pain referred into one or both legs that lasts for at least 1 day. Two parameters associated with LBP were measured: incidence, and DALYs. The incidence refers to the number of new cases of a given cause during a given period in a specified population. DALYs were derived by summing years of life lost and years lived with disabilities, thereby incorporating both premature death and health-related suffering to portray the total years of healthy life lost from all causes [7] DisMod-MR 2.1, a Bayesian meta-regression disease modeling tool, was used to estimate the prevalence and DALY, enforcing consistency between epidemiological parameters [4].

\section{Statistical analyses}

We calculated the EAPCs in the age-standardized rates of LBP. These EAPCs describe age-standardized rate trends within a specified time interval and were determined by the formula: $\mathrm{Y}=\alpha+\beta \mathrm{X}+\varepsilon$, where $\mathrm{Y}$ means $\ln$ (age-standardized rate), $\mathrm{X}$ is the calendar year, and $\varepsilon$ is the error term, $\beta$ refers to the positive or negative age-standardized rate trend.
Assuming that the natural logarithm of age-standardized rate is linear with time, therefore, $\operatorname{EAPC}=100 \times[\exp (\beta)-1]$. The $95 \%$ confidence interval (CI) of an EAPC was calculated from the linear model. When EAPC and its 95\% CI lower limit are both positive, its corresponding age-standardized rate shows an upward trend; contrastingly, when EAPC and its $95 \%$ CI upper limit are both negative, its corresponding age-standardized rate shows a downward trend. Otherwise, age-standardized rate is regarded as stabilized [8].

The correlations between the EAPCs and age-standardized rates and between the SDIs and EAPCs were calculated by using Gaussian process regression and Pearson's correlation coefficient $(\rho)$. All calculations were performed using R software (version 3.5.1).

\section{Results}

\section{Analysis of the LBP incidence}

From 1990 to 2019, the global incidence of LBP increased by $50 \%$, from $149,294,134.47$ to $223,455,640.82$ cases (Table 1). Consistently, the global age-standardized incidence rate showed a downward trend with an EAPC of -0.41 ( -0.46 to -0.36$)$, decreasing from $3,168.93$ to 2,748.90 per 100,000 persons (Table 1; Fig. 1A; Supplementary Fig. 1A). The age-standardized incidence rate decreased in both sexes; male subjects had an EAPC of - 0.41 (95\% CI, -0.46 to -0.37 ), and female subjects had an EAPC of -0.41 (95\% CI, -0.47 to -0.36 ) (Table 1; Fig. 1A). The age-standardized incidence rate of LBP from 1990 to 2019 in female subjects was higher than that in male subjects, as demonstrated by the male-to-female ratios of 0.78 and 0.77 , respectively (Table 1).

On the other hand, the age-standardized incidence rate of LBP was much higher in the regions with high SDI values than in regions with other SDI values (Table 1; Supplementary Fig. 1A). For example, in 2019, the highest agestandardized incidence rate of LBP, 3,126.59 per 100,000 population, was observed in the high SDI regions, followed by the high-middle SDI regions at 2,824.31 per 100,000 population. In contrast, the age-standardized incidence rate of LBP decreased the most in the low-middle SDI region, where the EAPC was the lowest at -0.52 (95\% CI, -0.59 to -0.44) (Table 1; Fig. 1A).

Meanwhile, the incidence of LBP was the highest in the 80-84 years age group globally. In addition, the incidence of LBP was highest in the 80-84 age group in the high, highmiddle, middle, and low-middle SDI regions (Supplementary Fig. 2). In 2019, the high SDI regions had the highest proportion of incident cases of LBP, $23.34 \%$, among the young people (age 15-49) (Supplementary Fig. 3A). Regionally, in 2019, the highest age-standardized incidence rates of 


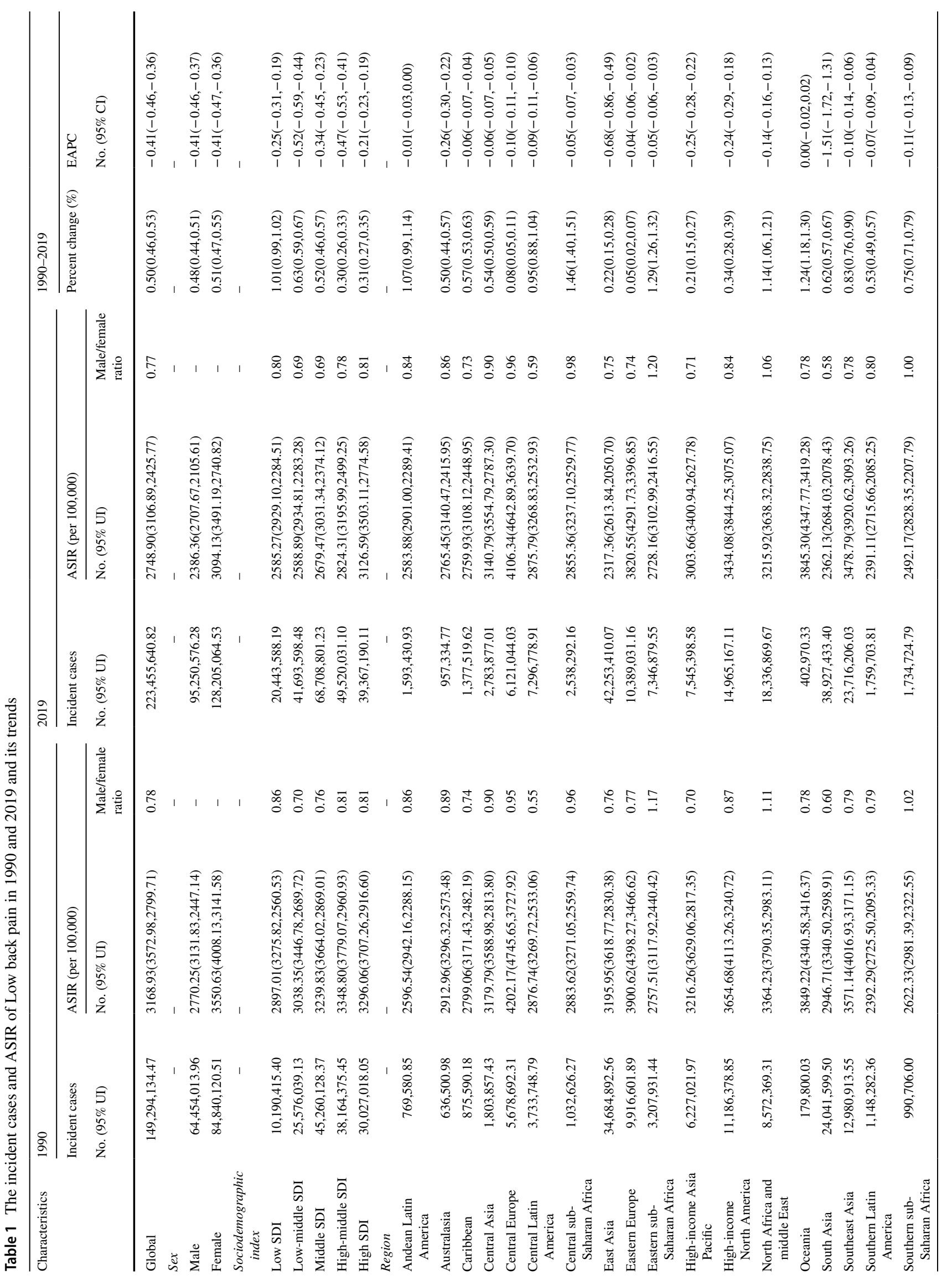




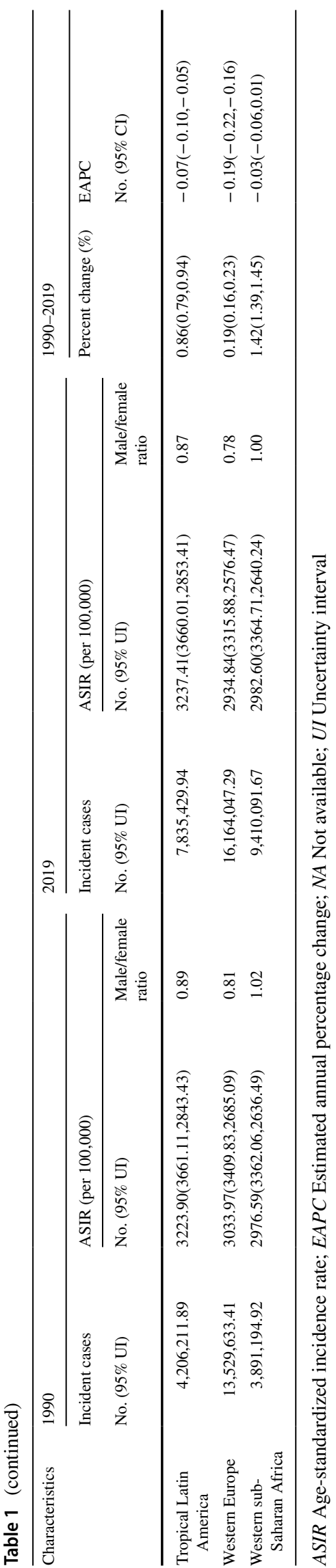

LBP were observed in Central Europe at 4,106.34, Oceania at 3,845.30, and Eastern Europe at 3,820.55, whereas the lowest age-standardized incidence rates of LBP were in East Asia at 2,317.36, South Asia at 2,362.13, and Southern Latin America at 2,391.11 per 100,000 population (Table 1). From 1990 to 2019, the age-standardized incidence rate of LBP decreased the most in South Asia with an EAPC of - 1.51 (95\% CI, -1.72 to -1.31 ), East Asia with an EAPC of - 0.68 (95\% CI, -0.86 to -0.49 ), and Australasia with an EAPC of -0.26 (95\% CI, -0.30 to -0.22 ) (Table 1; Fig. 1A).

At a country level, in 2019, the highest age-standardized incidence rates of LBP were observed in Poland (4,179.43 per 100,000 population), Vanuatu $(4,160.10)$, and Romania $(4,140.40)$, whereas the lowest age-standardized incidence rates of LBP were in India $(2,268.60)$, China $(2,280.67)$, and Singapore $(2,371.31)$ (Supplementary Table 1; Supplementary Table 3; Fig. 2A-C). From 1990 to 2019, the age-standardized incidence rate of LBP decreased the most in India (total: EAPC, -1.02 ; males: EAPC, -1.10 , females: EAPC, - 1.04) and increased the most in Zambia (total: EAPC, 0.18; males: EAPC, 0.17, females: EAPC, 0.19). (Supplementary Table 1; Supplementary Table 3; Fig. 3A-C).

\section{Analysis of LBP DALY worldwide}

From 1990 to 2019, the global DALYs of LBP increased by $47 \%$, from $43,361,648.41$ to $63,685,119.80$ (Table 2). In contrast, the age-standardized DALY rate displayed a downward trend, with an EAPC of $-0.51(-0.56$ to -0.46$)$ (Table 2; Fig. 1B). The age-standardized DALY rate decreased in both sexes, with an EAPC of -0.47 (95\% CI, -0.52 to -0.42$)$ in male subjects and an EAPC of -0.54 (95\% CI, -0.60 to -0.49 ) in female subjects (Table 2; Fig. 1B). The agestandardized DALY rate of LBP in females was higher than that in males, as demonstrated by the male-to-female ratios of 0.75 and 0.76 , respectively (Table 2 ).

On the other hand, the age-standardized DALY rate of LBP was much higher in regions with high SDI values than in regions with other SDI values (Table 2; Supplementary Fig. 1B). In 2019, the highest age-standardized DALY rate of LBP in the high SDI regions, $1,142.67$ per 100,000 population (95\% UI, 809.85 to $1,522.54$ ), was followed by that in the high-middle SDI regions at 773.88 per 100,000 population (95\% UI, 546.26 to $1,038.23)$. The age-standardized DALY rate of LBP decreased the most in the low-middle SDI region, where the EAPC was lowest at $-0.55(95 \%$ CI, -0.63 to -0.46 ) (Table 2; Fig. 1B). Globally, the LBP DALY rate was highest in the 80-84 age group. In addition, the LBP DALY rate was highest at the 75-79 group in the high SDI regions, highest in the 80-84 group in the low SDI regions (Supplementary Fig. 4). In 2019, the high SDI regions had the highest proportion of DALY cases of LBP 
Fig. 1 The EAPC of LBP agestandardized rates from 1990 to 2019, by sex and region. A The EAPC of age-standardized incidence rate. B The EAPC of age-standardized DALY rate. $\mathrm{EAPC}=$ estimated annual percentage change. DALY $=$ disability-adjusted life year
A age standardized incidence rate EAPC

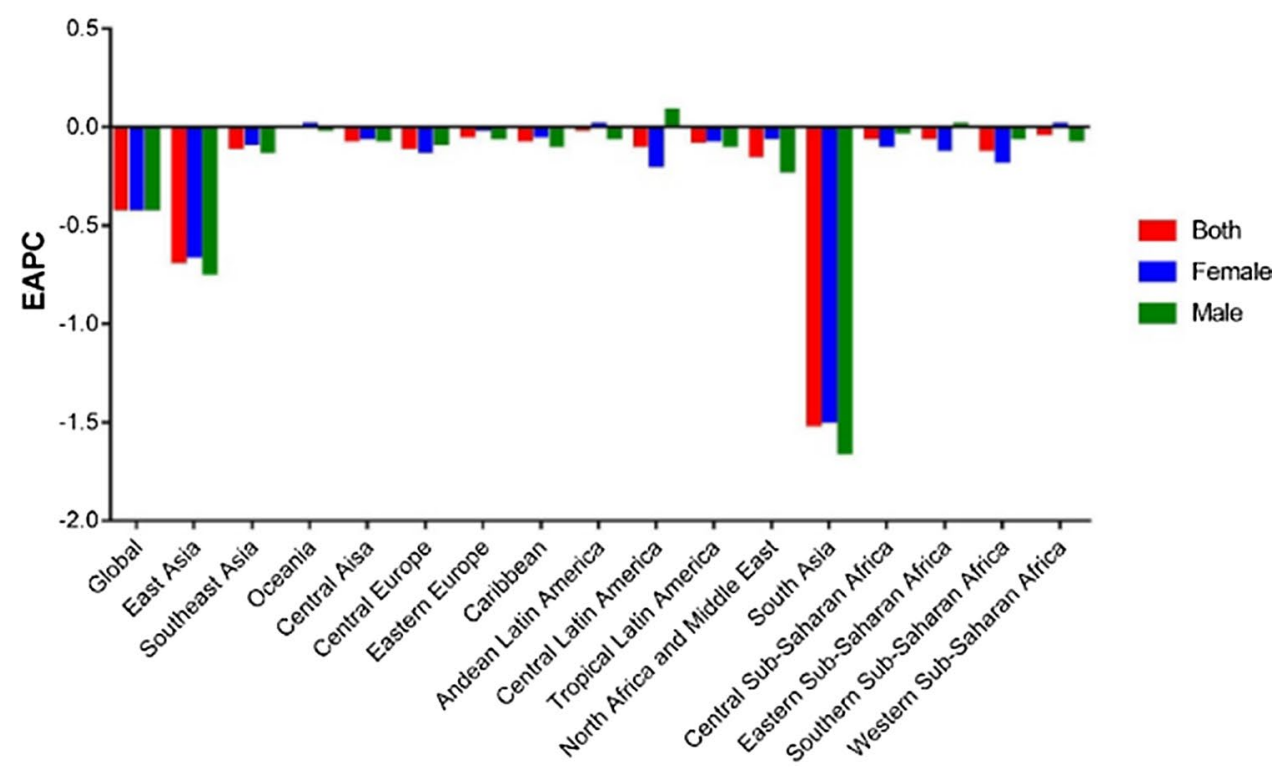

B age standardized DALY rate EAPC

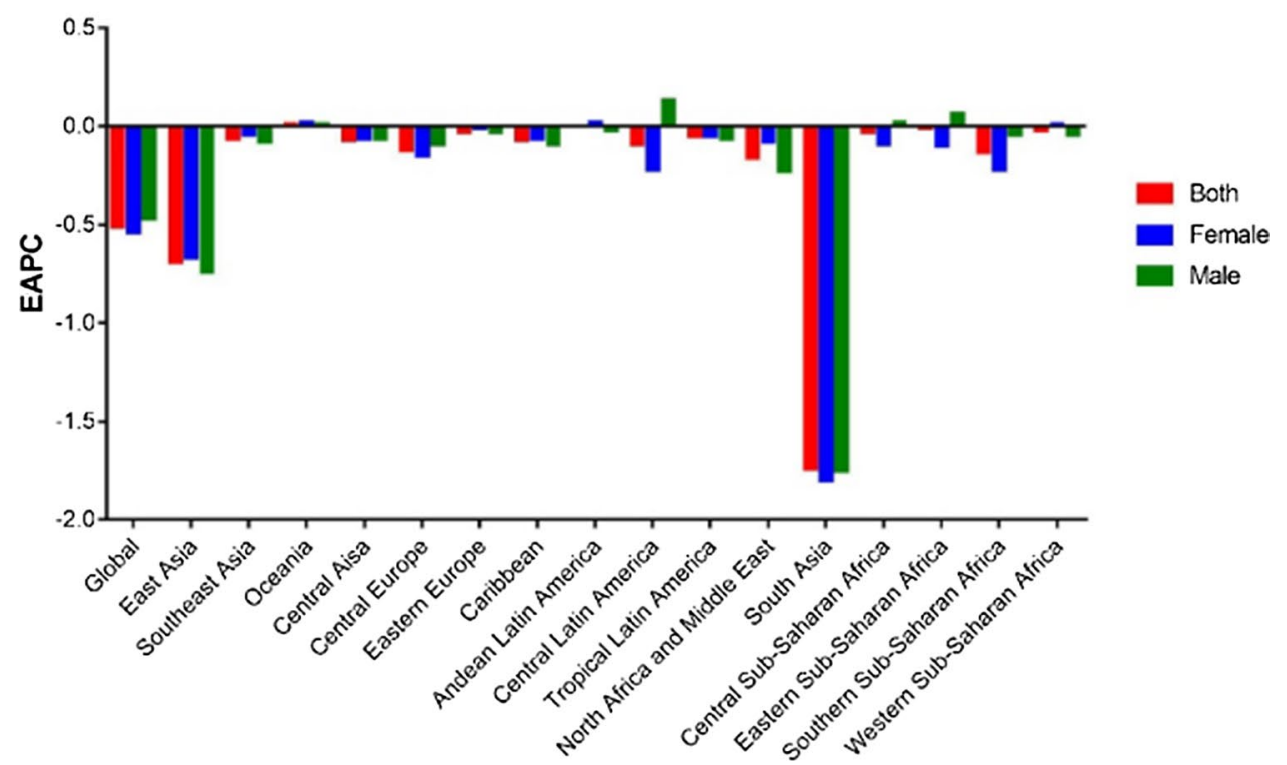

in young people (age 15-49), with a rate of $21.43 \%$ (Supplementary Fig. 3B).

At the regional level, in 2019, the highest age-standardized DALY rate of LBP was observed in high-income North America at 1,362.08 per 100,000 population, Central Europe at 1,127.05, and the high-income Asia-Pacific at 1080.52. Meanwhile, the lowest age-standardized DALY rate of LBP was observed in East Asia at 589.12 per 100,000 population, South Asia at 603.50, and Southern sub-Saharan Africa at 638.79 (Table 2). From 1990 to 2019, the age-standardized
DALY rate of LBP only increased in Oceania, with an EAPC of 0.01 (95\% CI, -0.01 to 0.03$)$, and decreased the most in South Asia, with an EAPC of - 1.74 (95\% CI, - 1.99 to -1.49$)$, East Asia with an EAPC of -0.69 (95\% CI, -0.89 to -0.50$)$, and Australasia with an EAPC of $-0.34(95 \%$ CI, -0.41 to -0.27 ) (Table 2; Fig. 1B).

At the country level, in 2019, the highest of LBP were observed in the United States at 1,402.94, Denmark at $1,248.54$, and Switzerland at $1,197.23$ per 100,000 population, whereas the lowest of LBP were observed in India at 
575.01, China at 579.05, and Eswatini at 614.68 per 100,000 population (Supplementary Table 1; Supplementary Table 4; Fig. 2D-F). From 1990 to 2019, the age-standardized DALY rate of LBP decreased the most in India (total EAPC: - 1.14; EAPC in males: - 1.15, EAPC in females: - 1.21) and increased the most in Zambia (total EAPC: 0.26; EAPC in males: 0.26, EAPC in females: 0.26) (Supplementary Table 3; Fig. 3 D-F).

\section{Discussion}

This study contributes to the understanding of the worldwide epidemiology of LBP by systematically analyzing the related disease burden using data from the GBD 2019 study. The global incidence of LBP and number of LBP-caused DALY cases significantly increased from 1990 to 2019. However, the age-standardized incidence rate and age-standardized DALY rate of LBP showed a global downward trend, and the age-standardized incidence rate decreased the most in South Asia, and East Asia. For countries, the largest decreases in the age-standardized incidence rate and age-standardized DALY rate of LBP were observed in India, and China.

In the context of socioeconomic development, the largest decreases in China and India might be due to the increasing awareness of health [9]. Besides, with the development of economy, the Chinese population was earning more and paying more attention to health, and young people preferred some light manual labor $[1,10,11]$. Also, due to the wellknown increasing economical and sociological pressure upon the peoples of India and China, the incidence of LBP might be under-reported in younger. Thus, the results in our study should be interpreted with caution.

In this study, the male/female ratio indicated a higher incidence and DALY rate among females [12-15]. Based on the current evidence, this finding may be attributed to multiple female-related characteristics, including a greater awareness of the symptoms and signs of diseases; potentially greater intensity of housework in non-ergonomic positions; more overload of the back due to anatomical and functional differences; and pregnancy-related physiological alterations $[12,16]$. Moreover, given that many factors are associated
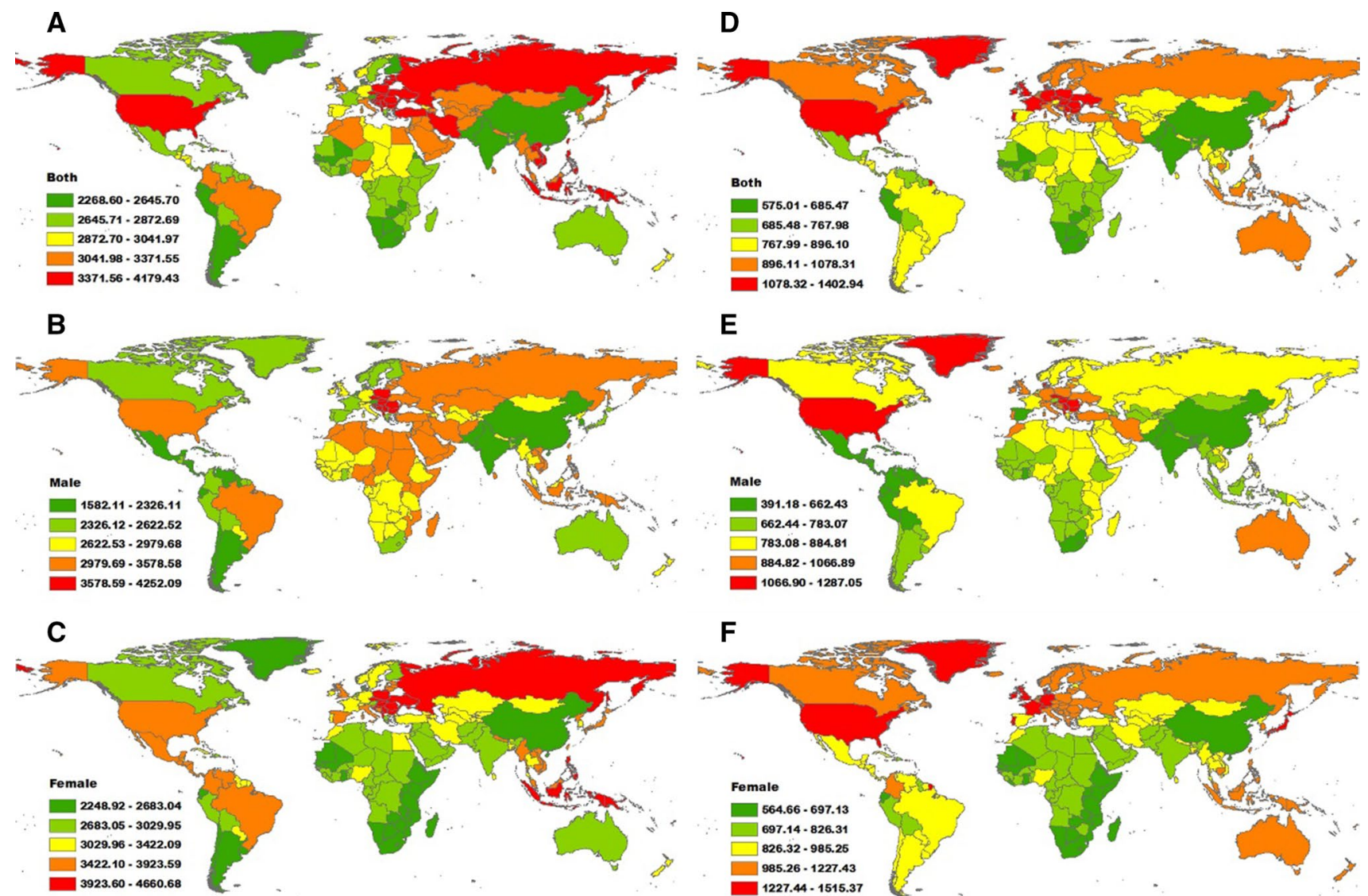

Fig. 2 The global age-standardized rates of LBP for both genders in 204 countries in 2019 , by countries. A The age-standardized incidence rate in both. $\mathbf{B}$ The age-standardized incidence rate in male. $\mathbf{C}$ The age-standardized incidence rate in female. $\mathbf{D}$ The age-standard-

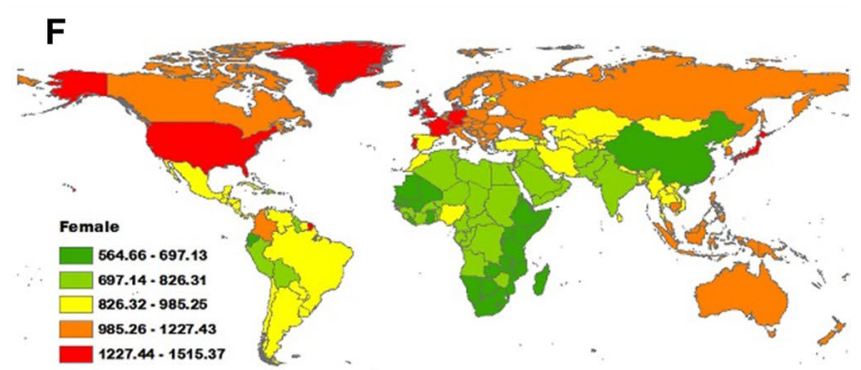

ized DALY rate in both. $\mathbf{E}$ The age-standardized DALY rate in male. F The age-standardized DALY rate in female. DALY = disabilityadjusted life year 

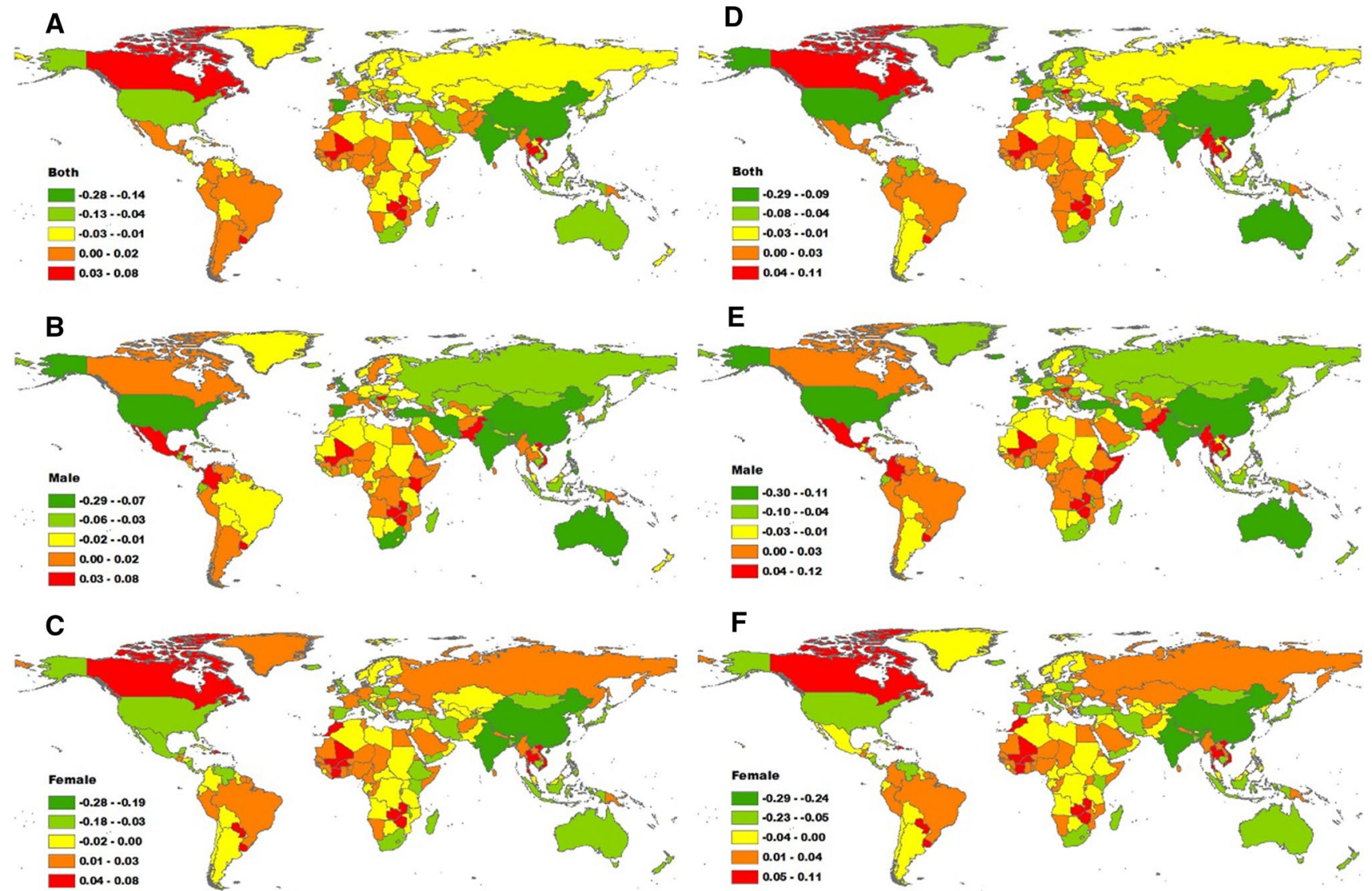

Fig. 3 The global EAPC of LBP age-standardized rates from 1990 to 2019, by countries. A The EAPC of ASIR in both. B The EAPC of ASIR in male. $\mathbf{C}$ The EAPC of ASIR in female. D The EAPC of agestandardized DALY rate in both. E The EAPC of age-standardized

with LBP and disability, sex-related differences in other biological, psychological, social, and societal factors may also contribute to this the higher DALY rate in females.

Meanwhile, population aging is expected to dramatically increase the economic and healthcare burdens due to musculoskeletal (MSK) conditions in the future [17]; this is important because most MSK conditions have mutual risk factors common to other prevalent chronic diseases associated with aging [18]. And LBP and its increasing disease burden are largely associated with population aging $[12,17]$. According to data from the GBD 2019 study, the age-standardized incidence rate and age-standardized DALY rate were observed in the 80-84 age group. In addition, the greatest number of people with LBP globally are in the 50-54 group [2]. These findings emphasize the importance of early prevention of and treatment intervention for LBP.

Our study offers the latest estimates of the burden of LBP at the global, regional, and national levels during the 1990-2019 period. Based on the analytical strategy used in the GBD 2019 study, extensive data from 204 countries and territories were used to measure the temporal trends of the
DALY rate in male. $\mathbf{F}$ The EAPC of age-standardized DALY rate in female. $\mathrm{EAPC}=$ estimated annual percentage change. age-standardized rates $=$ age-standardized rates. ASIR $=$ age-standardized incidence rate. $\mathrm{DALY}=$ disability-adjusted life year

age-standardized prevalence and DALYs of LBP. However, several inherent limitations of this study should be considered. First, self-reported LBP is mainly dependent on an individual's perception of the degree and frequency of signs and symptoms. In the GBD study, this limitation was mitigated by adjustment for variations in the recall period, anatomical location, minimum episode duration, and the extent to which the condition limits activity [12]. Second, our study is restricted to the previous described limitations of the GBD methods $[7,19,20]$. Despite the best efforts to collect all published and unpublished data, the quantity and quality of the current data remain limited. However, the GBD study used complex modeling to mitigate these data limitations and obtain more accurate estimates.

\section{Conclusion}

The global age-standardized incidence rate and age-standardized DALY rate of LBP showed a downward trend, especially in East and South Asia. In addition, a heavier burden 


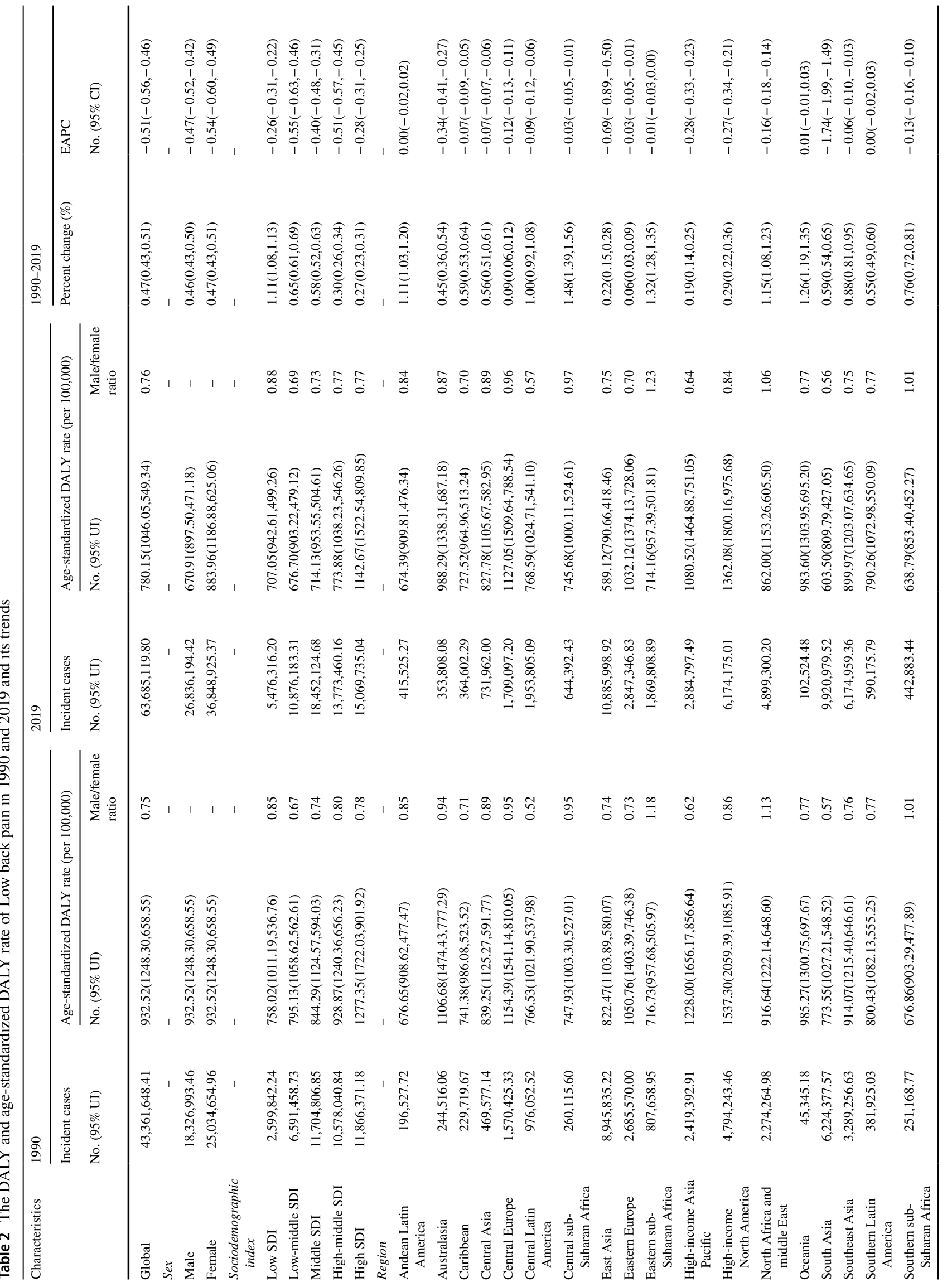


of LBP was observed in older and female populations. Therefore, preventive strategies for LBP, with a particular focus on older and female populations, can be crucial for the prevention of LBP.

Supplementary Information The online version contains supplementary material available at https://doi.org/10.1007/s00586-022-07133-x.

Authors' contributions XJC, LFW, and HY designed the study. LFW and $\mathrm{HY}$ and performed the initial research and writing of the first draft. XJC, JY, and MXL provided subject matter expertise. XJC, LFW, HY, ZCL, CWL, JY, and MXL all participated in final writing and editing of the manuscript.

Funding This study was reported by Fujian Provincial Natural Science Foundation of China (Grant number: 2020J011307).

\section{Declarations}

Conflict of Interest The authors declared that they have no conflict of Interest.

Open Access This article is licensed under a Creative Commons Attribution 4.0 International License, which permits use, sharing, adaptation, distribution and reproduction in any medium or format, as long as you give appropriate credit to the original author(s) and the source, provide a link to the Creative Commons licence, and indicate if changes were made. The images or other third party material in this article are included in the article's Creative Commons licence, unless indicated otherwise in a credit line to the material. If material is not included in the article's Creative Commons licence and your intended use is not permitted by statutory regulation or exceeds the permitted use, you will need to obtain permission directly from the copyright holder. To view a copy of this licence, visit http://creativecommons.org/licenses/by/4.0/.

\section{References}

1. Knezevic NN, Candido KD, Vlaeyen JWS, Van Zundert J, Cohen SP (2021) Low back pain. Lancet 398(10294):78-92. https://doi. org/10.1016/s0140-6736(21)00733-9

2. Vos T, Lim SS, Abbafati C, Abbas KM, Abbasi M, Abbasifard M, Abbasi-Kangevari M, Abbastabar H, Abd-Allah F, Abdelalim A, Abdollahi M (2020) Global burden of 369 diseases and injuries in 204 countries and territories, 1990-2019: a systematic analysis for the Global Burden of Disease Study 2019. Lancet 396(10258):1204-1222. https://doi.org/10.1016/s0140-6736(20) 30925-9

3. Kahere M, Ginindza T (2020) Mapping evidence on the prevalence, incidence, risk factors and cost associated with chronic low back pain among adults in Sub-Saharan Africa: a systematic scoping review protocol. Syst Rev 9(1):57. https://doi.org/10.1186/ s13643-020-01321-w

4. Roberts NL, Mountjoy-Venning WC, Anjomshoa M, Banoub JA, Yasin YJ (2018) Global, regional, and national incidence, prevalence, and years lived with disability for 354 diseases and injuries for 195 countries and territories, 1990-2017: a systematic analysis for the Global Burden of Disease Study 2017. Lancet 392(10159):1789-1858. https://doi.org/10.1016/s0140-6736(18) 32279-7

5. Stevens GA, Alkema L, Black RE et al (2016) Guidelines for accurate and transparent health estimates reporting: the GATHER 
statement. Lancet 388(10062):e19-e23. https://doi.org/10.1016/ s0140-6736(16)30388-9

6. http://ghdx.healthdata.org/gbd-2019. Accessed August 17, 2021.

7. Kyu HH, Abate D, Abate KH, Abay SM, Abbafati C, Abbasi N, Abbastabar H, Abd-Allah F, Abdela J, Abdelalim A, Abdollahpour I (2018) Global, regional, and national disability-adjusted life-years (DALYs) for 359 diseases and injuries and healthy life expectancy (HALE) for 195 countries and territories, 1990-2017: a systematic analysis for the Global Burden of Disease Study 2017. Lancet 392(10159):1859-1922. https://doi.org/10.1016/ s0140-6736(18)32335-3

8. Liu X, Jiang J, Yu C et al (2019) Secular trends in incidence and mortality of bladder cancer in China, 1990-2017: a joinpoint and age-period-cohort analysis. Cancer Epidemiol 61:95-103. https:// doi.org/10.1016/j.canep.2019.05.011

9. Fernandes JC (2018) Global, regional, and national age-sexspecific mortality and life expectancy, 1950-2017: a systematic analysis for the Global Burden of Disease Study 2017. Lancet 392(10159):1684-1735. https://doi.org/10.1016/s0140-6736(18) 31891-9

10. Collaborators GB, Ärnlöv J (2020) Global age-sex-specific fertility, mortality, healthy life expectancy (HALE), and population estimates in 204 countries and territories, 1950-2019: a comprehensive demographic analysis for the Global Burden of Disease Study 2019. Lancet 396(10258):1160-1203. https://doi.org/10. 1016/s0140-6736(20)30977-6

11. Wu A, Dong W, Liu S et al (2019) The prevalence and years lived with disability caused by low back pain in China, 1990 to 2016: findings from the global burden of disease study 2016. Pain 160(1):237-245. https://doi.org/10.1097/j.pain.0000000000 001396

12. de David CN, Deligne LMC, da Silva RS et al (2020) The burden of low back pain in Brazil: estimates from the Global Burden of Disease 2017 Study. Popul Health Metr 18(Suppl 1):12. https:// doi.org/10.1186/s12963-020-00205-4

13. Malta DC, Oliveira MM, Andrade S, Caiaffa WT, Souza MFM, Bernal RTI (2017) Factors associated with chronic back pain in adults in Brazil. Rev Saude Publica 51(suppl 1):9s. https://doi.org/ 10.1590/s1518-8787.2017051000052
14. Maher C, Underwood M, Buchbinder R (2017) Non-specific low back pain. Lancet 389(10070):736-747. https://doi.org/10.1016/ s0140-6736(16)30970-9

15. Iguti AM, Bastos TF, Barros MB (2015) Back pain in adults: a population-based study in Campinas, São Paulo State, Brazil. Cad Saude Publica 31(12):2546-2558. https://doi.org/10.1590/0102$311 \times 00178114$

16. Ferreira ML, Machado G, Latimer J, Maher C, Ferreira PH, Smeets RJ (2010) Factors defining care-seeking in low back paina meta-analysis of population based surveys. Eur J Pain 14(7):747. e1-7. https://doi.org/10.1016/j.ejpain.2009.11.005

17. Hoy DG, Smith E, Cross M et al (2015) Reflecting on the global burden of musculoskeletal conditions: lessons learnt from the global burden of disease 2010 study and the next steps forward. Ann Rheum Dis 74(1):4-7. https://doi.org/10.1136/annrh eumdis-2014-205393

18. Briggs AM, Woolf AD, Dreinhöfer K et al (2018) Reducing the global burden of musculoskeletal conditions. Bull World Health Organ 96(5):366-368. https://doi.org/10.2471/blt.17.204891

19. Murray C, Callender CS, Kulikoff R, Srinivasan V, Abate D, Abate K, Lim S (2018) Population and fertility by age and sex for 195 countries and territories, 1950-2017: a systematic analysis for the Global Burden of Disease Study 2017. Lancet 392(10159):1995-2051. https://doi.org/10.1016/s0140-6736(18) 32278-5

20. Global CG (2018) Global, regional, and national comparative risk assessment of 84 behavioural, environmental and occupational, and metabolic risks or clusters of risks for 195 countries and territories, 1990-2017: a systematic analysis for the Global Burden of Disease Study 2017. Lancet 392(10159):1923-1994. https:// doi.org/10.1016/s0140-6736(18)32225-6

Publisher's Note Springer Nature remains neutral with regard to jurisdictional claims in published maps and institutional affiliations. 